\title{
The Construction of the Dupin Cyclides in a Smectic A Polygonal Texture
}

\author{
Claire Meyer, Brice Jonckheere, and Coline Penaud \\ Laboratoire de Physique des Systèmes Complexes, Université de Picardie Jules Verne, 33 rue Saint-Leu, 80039 Amiens, France \\ Correspondence should be addressed to Claire Meyer; claire.meyer@u-picardie.fr
}

Received 28 January 2014; Accepted 22 February 2014; Published 30 March 2014

Academic Editor: Rodrigo Martins

Copyright ( 2014 Claire Meyer et al. This is an open access article distributed under the Creative Commons Attribution License, which permits unrestricted use, distribution, and reproduction in any medium, provided the original work is properly cited.

We report experimental observations with optical microscopy of the usual so-called polygonal texture. We have made simulations of the Dupin cyclides in one small region of the sample.

\section{Introduction}

Many experiments on smectic A phases of liquid crystals report on the existence of macroscopic defective textures since the pioneer work of Friedel and Grandjean of 1910 [1]. Such a liquid crystal phase put in evidence an interconnection between physics and mathematics as nicely illustrated in [2]. Because of the nature of the singular lines in a smectic phase, two confocal lines (ellipse and hyperbola or two confocal parabolas) both located in perpendicular plans, smectic layers take the shape of Dupin cyclides in order to keep their equidistance. Notice that other defective lines, the double helices, exist also in the smectic A phase of a liquid crystal. They were first reported by Williams [3] and the smectic layers at their vicinity take the form of ruled helicoids [4]. Let us focus in this paper on the FCD textures that we describe here. The cover of [5] shows a nice polygonal domain, notion first introduced by Friedel [6]. This texture is highly regular and presents different properties. Figure 1 shows the same kind of texture. We can distinguish two different networks of polygons while focusing on the bottom of the substrate (Figure 1(a)) or on the top (Figure 1(b)) for a $75 \mu \mathrm{m}$ sample thickness.

Let us focus on a part of the ellipses network, which appear inside the white frame (see Figure 1(a)). Figure 2(a) shows a zoom of one part of the experimental network of the sample and Figure 2(b) shows the simulation of the only several ellipses: the biggest one and the other six, which are in contact with the biggest one. One of the ellipses being too small, we will not take this ellipse into account in what follows. For this simulation, we first assume that the biggest ellipse is in the plane of the top of the substrate. The six other ellipses are drawing after the realization of a small rotation of different angles in the plane of the bottom of the substrate.

After measuring different parameters like semimajor and semiminor axis for the seven ellipses and the distances between apices from the experimental observations, we try to give an overview of how the smectic layers in such a sample are keeping in mind that adjacent layers coming from two different FCDs should meet continuously. We use the Image J software, which permits us to measure directly from the optical crossed polarizers picture the values of these parameters and also of the different angles $\theta_{F_{i}}$ between the semimajor axis of each ellipse $i$ with respect to the biggest one (called ellipse 0 ) measured from its physical focus $F_{0}$ (in blue in Figure 2(b)). Figure 2(b) shows also that $\theta_{F_{3}}=0$. By using one translation and a rotation matrix of angle $\theta_{F_{i}}$ in the plane of Figure 2 and also a size reduction, we were able to find how each ellipse $i$ can be obtained from the biggest one (numbered 0 ). What is interesting in this approach is the fact that we only use the measurement of the position of the physical focus $F_{0}$ of the biggest ellipse 0 and there is no need to take into account the positions of the other physical foci for all other ellipses $i$, which are less well defined already due to their smaller size.

Before thinking about the construction of the layers, let us begin to recall the usual description of the smectic layers at the vicinity of two different focal lines made with an ellipse 


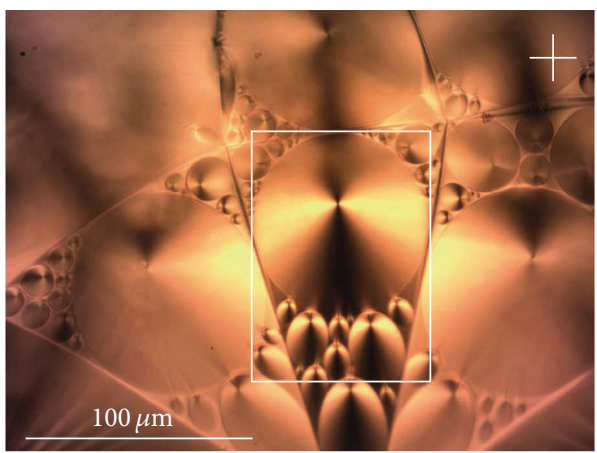

(a)

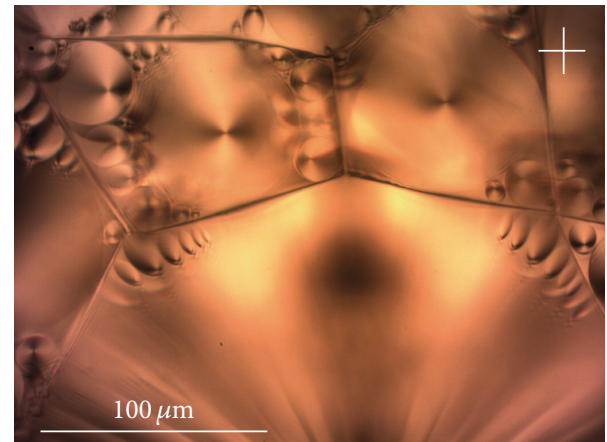

(b)

FIGURE 1: Observation with crossed polarizers optical microscope of the polygonal texture with two different focusing: (a) on the bottom of the substrate and (b) on the top, $75 \mu \mathrm{m}$ sample thickness.

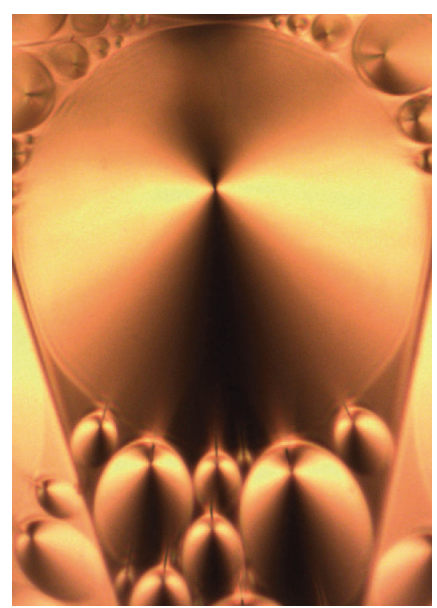

(a)

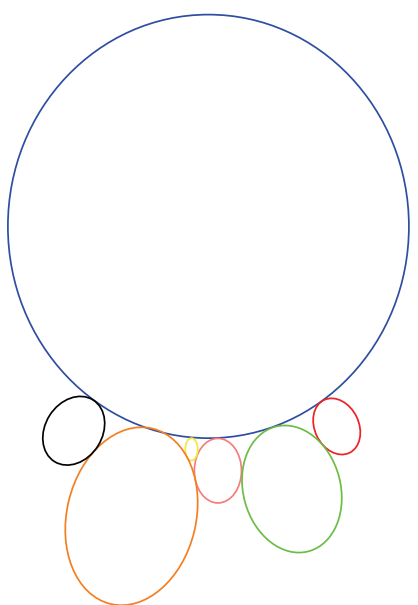

(b)

FIGURE 2: Investigated part of the sample observed under optical crossed polarizers: (a) zoom of a part of Figure 1 and (b) simulation of seven ellipses.

$E$ of semimajor and semiminor axes $a$ and $b$, respectively; the distance from the center of the ellipse to the focus is called $c=\sqrt{a^{2}-b^{2}}$ and with the confocal hyperbola $H$. The two lines $E$ and $H$ are in two perpendicular planes and their equations are written as follows:

$$
\begin{gathered}
E\left\{\begin{array}{c}
x=a \cos u, \\
y=b \sin u, \\
z=0,
\end{array}\right. \\
H\left\{\begin{array}{c}
x=c \cosh v, \\
y=0, \\
z=b \sinh v,
\end{array}\right.
\end{gathered}
$$

where $u \in[0,2 \pi]$ and $v \in\left[-v_{\max }, v_{\max }\right] ; v_{\max }$ is chosen in the locations where the hyperbola physically stops in the sample. Here, we assume in fact that the maximum and minimal values of $v$ are symmetric, whereas it is usually not the case, but it does not change our discussion here. The points where the hyperbolae meet in a sample have been called "poles" by
Friedel [6]. The segment, which joins a point $M$ of the ellipse $E$ (1) and a point $P$ of the hyperbola $H(1)$, is normal to a Dupin cyclide (2) (see [7]). The equation of Dupin cyclides surfaces is indeed given as follows:

$$
\overrightarrow{\mathrm{OM}}\left\{\begin{array}{c}
x=\frac{\mu(c-a \cos u \cos v)+b^{2} \cos u}{a-c \cos u \cos v}, \\
y=\frac{b \sin u(a-\mu \cos v)}{a-c \cos u \cos v}, \\
z=\frac{b \sin v(c \cos u-\mu)}{a-c \cos u \cos v},
\end{array}\right.
$$

where $u$ and $v$ correspond to the surface parameters and each surface, that is, each Dupin cyclide, corresponds to a fixed value of $\mu$. With derivatives calculations and the first two fundamental forms, we find from (2) the following two 
different main curvature radii (see the Appendix for a detailed calculation):

$$
\begin{aligned}
& R_{1}=\frac{1}{\rho_{1}}=\varepsilon(\mu-a \sec v), \\
& R_{2}=\frac{1}{\rho_{2}}=\varepsilon(\mu-c \cos u) .
\end{aligned}
$$

In the particular case where these two numbers are equal (double root), the corresponding points are called umbilics. The strengths of these umbilical defects $( \pm 1)$ are often encountered in nematic liquid crystals with a higher velocity for the positive one [8].

The Gaussian curvature $G$ (also called total curvature) and the mean curvature can be, respectively, written as

$$
\begin{gathered}
G=\frac{1}{R_{1} R_{2}}=\frac{1}{(\mu-a \sec v)(\mu-c \cos u)}, \\
H=\frac{1}{\varepsilon(\mu-a \sec v)}+\frac{1}{\varepsilon(\mu-c \cos u)} .
\end{gathered}
$$

Note that the case of a torus is simply obtained replacing $c$ by zero in the expression of $R_{2}$.

Figure 3 illustrates one smectic layer for a Toroidal Focal Conic Domain (TFCD) (Figure 3(a)) and for a Focal Conic Domain (Figure 3(b)). The FCD is made by an ellipse and a hyperbola whereas the TFCD corresponds to the case where the ellipse is degenerated to a circle and the hyperbola to a straight line. The equations of the two different sets of singular lines are given in (1) for the ellipse and the confocal hyperbola and in (6) for the circle and the straight line going through the center of the circle:

$$
\begin{array}{cl}
x=a \cos u, & y=a \sin u, \quad z=0, \\
x=0, & y=0, \quad z=v,
\end{array}
$$

where $v$ varies from $v_{\min }$ to $v_{\max }$.

Taking any point $M$ of the surface, two different perpendicular plans in $M$ contain the normal at $M$ to the surface. These two plans intersect the surface into two different curvature lines, which are circles for both surfaces (torus and Dupin cyclides). These two circles of radii $R_{1}$ and $R_{2}$, respectively, are represented in green in Figure 3.

Applying (3) and (4), we obtain the different main curvatures values taking $v=0$ and $u=0$ corresponding to the chosen point $M$, for the torus:

$$
\begin{aligned}
& R_{1}(\text { torus })=\mu-a / \cos v=\mu-a=4-5=-1 \text { and } \\
& R_{2} \text { (torus) }=\mu-c \cos u=\mu-c=4-0=4,
\end{aligned}
$$

and for the Dupin cyclide:

$$
\begin{aligned}
& R_{1}(\text { cyclide })=\mu-a / \cos v=\mu-a=4-5=-1 \text { and } \\
& R_{2}(\text { cyclide })=\mu-c \cos u=\mu-c=4-3=1 \text { (note that } \\
& \text { the two main curvature lines being circles, which are } \\
& \text { planar curves, the main curvature radii of these lines } \\
& \text { can be positive or negative). }
\end{aligned}
$$

By the way, let us mention that the mean curvature $H$ for the Dupin cyclide is equal to 0 .

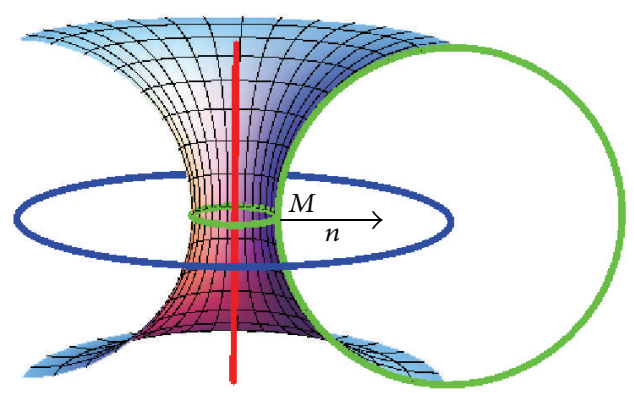

(a)

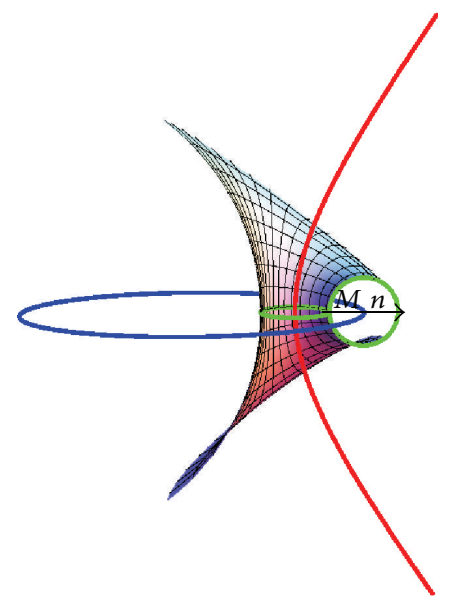

(b)

FIgure 3: Two different main curvature radii for two surfaces: a torus and a Dupin cyclide, both characterized by the same value of $\mu$ ( $\mu=4$ for the drawing). For the figure, $a=5$ and $b=4$. The two circles correspond to the curvature lines (in green) of radii $R_{1}$ and $R_{2}$, respectively, at any point $M$ of each surface; (a) $R_{1}$ (torus) $=-1$ and $R_{2}$ (torus) $=4$ and (b) $R_{1}($ cyclide $)=-1$ and $R_{2}($ cyclide $)=1$.

From (4), we see that the Gaussian curvature at $M$ is negative for the two surfaces. Also, we can remark that one of the main curvature lines is mingled with the generator circle of the torus but the second main curvature line is not mingled with the director circle represented in blue in Figure 3(a) [9].

Now, in order to build our layered system for the polygonal texture, we have to keep in mind that a focal domain possesses boundaries. The hyperbola stops in two poles and therefore two different cones exist even if only one cone whose apex is on the pole has been represented in Figure 4. Several layers existing inside the area defined by this cone have been drawn and one can distinguish the three different kinds of layers (type 1 when the Dupin cyclides are singular on the ellipse, type 3 when the Dupin cyclides are singular on the hyperbola, and type 2 when the Dupin cyclides do not meet the singular lines) [9].

For all the ellipses visible in the polygonal texture, we have to build the layers inside the different FCDs. One additional law named the law of corresponding cones is important [7]. The different ellipses are in contact one to each other in one point and at this point the generators of the cones associated with the two adjacent FCDs are the same. In our tiling, we have made the simulation of the different ellipses making 


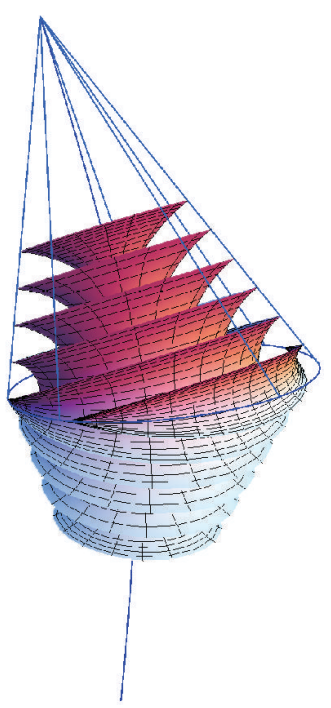

(a)

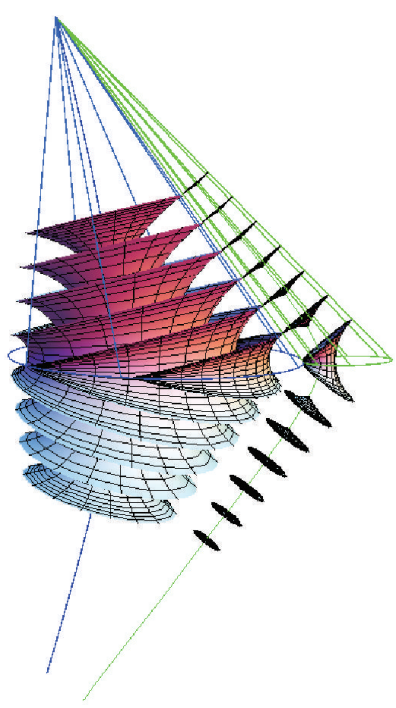

(b)

FIgURE 4: Several layers have been represented including inside Focal Conic Domains: (a) isolated FCD and (b) law of corresponding cones for two adjacent FCDs.

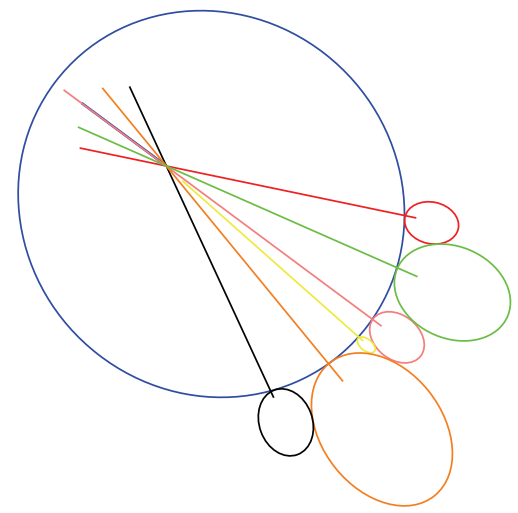

Figure 5: Representation of the top view of the seven ellipses and their confocal hyperbolae, which merge towards a small space region.

the hypothesis that they are all in contact, and after finding a semiempirical approach consisting of two steps, rotation and translation and size reduction of the bigger ellipse and then the direct measurement of the sizes of the ellipses via the software ImageJ, we obtain the tiling of ellipses with their confocal hyperbolae of Figure 5. Because we know how each ellipse $i$ is built from ellipse 0 using a translation, a rotation, and a size reduction, we can build the Dupin cyclides for all the ellipses using exactly the same transformations but in the $3 \mathrm{D}$ space. The hyperbolae have to meet all in one point but the simulations do not give enough precision to be able to find a single point; they converge towards a zone larger than a point even if it is not visible with the top view of Figure 5.

Now, we represent the different FCD associated with six ellipses and their confocal hyperbolae: one of the ellipses being too small (the yellow one). This yields to Figure 6 where a tiling has been made using Mathematica 8.0. The first remark is that the quasi-continuity of the layers is realized so that we are able to go continuously from one layer to the adjacent one. To ensure the continuity of the layers, we have to write (3) for two adjacent layers: one belonging to the layers of the FCD0 (relative to ellipse 0) and the other layer belonging to the layers of the FCD5 (relative to ellipse 5). One of the two radii of curvature is positive whereas the adjacent one is negative so that we get the following:

$$
\left(\mu_{0}-a_{0} \sec v\right)=-\left(\mu_{5}-a_{5} \sec v\right) .
$$

We therefore obtain the value of $\sec v$ then the value of $v$ for all the different locations where cyclides of adjacent FCDs meet. For example, if we take the upper drawn layer of ellipse 0 and the upper corresponding one of ellipse 5, we obtain a value of $v \cong 61^{\circ}$; then, the layer drawn just below corresponds to $v \cong 54^{\circ}$; then, for the layer drawn just below again, $v \cong$ $42^{\circ}, \ldots$

Among Friedel's association law, two FCDs cannot recover themselves; this argument shows that we can slightly change parameters in order that this law is better obeyed for the layers above the median plane containing approximately all the ellipses. This is clearly visible in Figure 4(b) where the layers below this median plane do not join correctly whereas they meet correctly above this same plane. In particular, our simulation permits us to slightly tilt each ellipse $i$ of a small angle (less than $2^{\circ}$ ) to permit the layers to continuously meet. There are therefore locations where Friedel's laws are no more completely obeyed. In [10], a model suggests a geometrical way of extending Friedel's law to fill the entire space of the sample with portion of Dupin cyclides and spheres, joining together not only the FCDs within a polygonal domain but also between two neighboring polygonal domains. This model has been nicely illustrated in Figure 14 of [11] in the case of a SmA droplet suspended in a glycerin matrix with the family of FCDs and interstices filled with spherical layers. In fact, inside a spherical droplet, we can see several circles of various sizes of radii $\rho$ on the surface of this spherical 


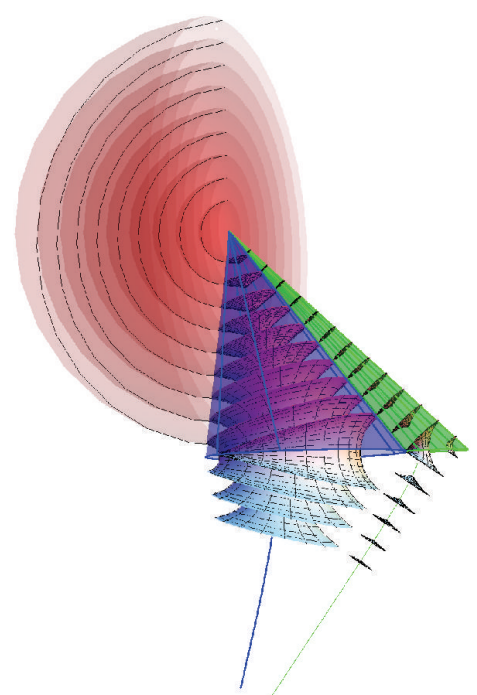

(a)

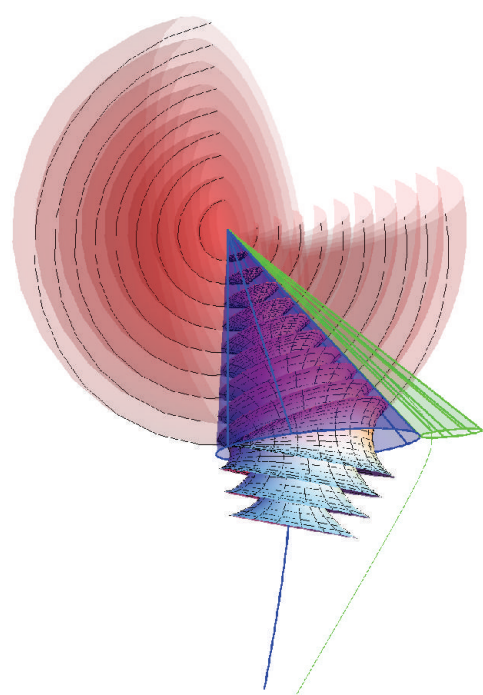

(b)

FIGURE 6: Two ellipses belonging to the polygonal domain and their corresponding cones: (a) $\rho>\rho^{*}$ green cone filled by FCDs and (b) $\rho<\rho^{*}$ green cone filled by parts of spheres.

droplet. These circles form an Apollonius spherical packing. Two cases are to be considered.

(i) When the radius of the circles $\rho$ is $>$ a certain value called $\rho^{*}$, then these regions are filled by layers taking the form of Dupin cyclides.

(ii) When the radius of the circles $\rho<\rho^{*}$, then these interstitial regions are filled by layers taking the form of concentric spheres. The presence of Dupin cyclides layers permits us to reduce the free energy density because of the negative Gaussian curvature instead of a positive one for spherical layers. In [10], the critical value of the circle size is $\rho^{*} \sim R / 10$ where $R$ denotes the radius of the droplet. In our situation, if $R$ denotes the typical size of a polygonal domain, we are in good agreement with this estimation: the size $R \sim 100$ microns and the $\rho^{*}$ can be evaluated to be close to about 10 microns.

Figure 6 represents the case of only two ellipses belonging to the polygonal domain and their corresponding cones. Figure 6(a) corresponds to the case where $\rho>\rho^{*}$ so that inside the green cone FCDs have been drawn, whereas Figure 6(b) corresponds to the case where $\rho<\rho^{*}$ so that inside the green cone part of spheres has been drawn.

The presence of such concentric spheres has been emphasized in the construction of the defects and demonstrated using topology in [12]. Hence, two different regions exist: one made with FCD and another one made with parts of spheres for the smallest circles.

To fill entirely the space, we have therefore to consider not only Dupin cyclides inside FCDs but also part of spheres in the interstitial regions. Figure 7 constitutes therefore just a picture to imagine how the layers could be in this tiling in first approximation. We have to keep in mind that the parts of spheres for the smallest interstitial regions have not been represented in Figure 7. In particular, the layers corresponding to the smallest ellipse (ellipse 4) should be parts of spheres because the cone of apex $S$ which lies on the ellipse 4 intercepts the spheres of center $S$ in very small circles.

\section{Conclusion}

We have presented a picture of the smectic layers inside a sample made with a polygonal texture. Before, for instance, the addition of nanoparticles inside a smectic phase, we should know as precisely as we can how the 3D layers are arranged inside a sample. Recently, a pillar assisted Epitaxial Assembly of TFCD in a SmA liquid crystal has been reported and presents a very regular network of TFCD [13]. In another paper, the authors imagine a construction of an indented square pyramid around each pillar (in the case of four FCDs around a circular pillar) and the corresponding hyperbolas intersect at the pyramid's apex along with portion of concentric spheres [14]. This kind of questions is very interesting and in the future we can think of using the polygonal texture for including nanoparticles in a texture which is less regular than a square set of FCD but nevertheless well ordered. Notice that an important and nice work in this field has been already done in [15].

\section{Appendix}

Calculation of the two curvature radii of a Dupin cyclide.

Making the calculation of the first derivatives of $\overrightarrow{O M}$ relating to $u$ and $v$, respectively, we get the following equation:

$$
\begin{aligned}
& \frac{\partial x}{\partial u}=\frac{b^{2} \sin u(\mu \cos v-a)}{(a-c \cos u \cos v)^{2}} \\
& \frac{\partial y}{\partial u}=\frac{b(a-\mu \cos v)(a \cos u-c \cos v)}{(a-c \cos u \cos v)^{2}}
\end{aligned}
$$




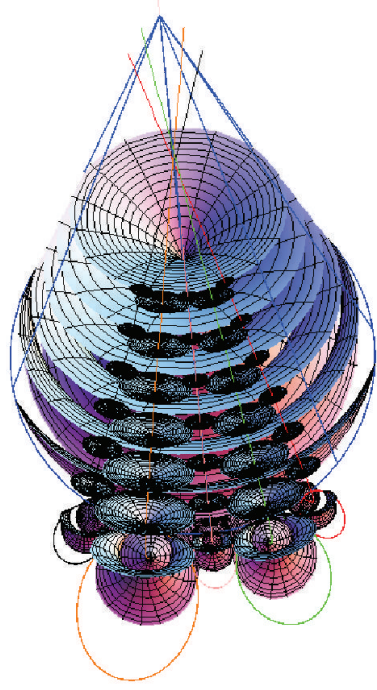

(a)

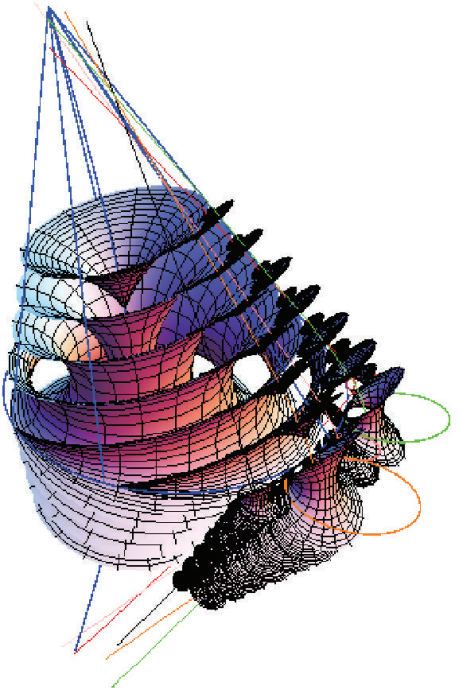

(b)

FIGURE 7: Simulation of the polygonal texture only on a small region: (a) top view and (b) side view.

$$
\begin{aligned}
& \frac{\partial z}{\partial u}=\frac{b \sin v c \sin u(\mu \cos v-a)}{(a-c \cos u \cos v)^{2}}, \\
& \frac{\partial x}{\partial v}=\frac{b(\mu-c \cos u)}{(a-c \cos u \cos v)^{2}} b \cos u \sin v, \\
& \frac{\partial y}{\partial v}=\frac{b(\mu-c \cos u)}{(a-c \cos u \cos v)^{2}} a \sin u \sin v, \\
& \frac{\partial z}{\partial v}=\frac{b(\mu-c \cos u)}{(a-c \cos u \cos v)^{2}}(c \cos u-a \cos v) .
\end{aligned}
$$

These equations yield to the expression of the three coefficients existing in the expression of the first fundamental quadratic form:

$$
\varphi_{1}^{M}(d M)=E d u^{2}+2 F d u d v+G d v^{2}
$$

where $E, F$, and $G$ are written as (see the following equation)

$$
\begin{gathered}
E=\left\|\frac{\partial \vec{M}}{\partial u}\right\|^{2}=\frac{b^{2}(\mu \cos v-a)^{2}}{(a-c \cos u \cos v)^{2}}, \\
F=\frac{\partial \vec{M}}{\partial u} \cdot \frac{\partial \vec{M}}{\partial v}=0, \\
G=\left\|\frac{\partial \vec{M}}{\partial v}\right\|^{2}=\frac{b^{2}(\mu-c \cos u)^{2}}{(a-c \cos u \cos v)^{2}} .
\end{gathered}
$$

The first quadratic fundamental form reads now as

$$
\begin{aligned}
\varphi_{1}^{M}(d M)= & \frac{b^{2}(\mu \cos v-a)^{2}}{(a-c \cos u \cos v)^{2}} d u^{2} \\
& +\frac{b^{2}(\mu-c \cos u)^{2}}{(a-c \cos u \cos v)^{2}} d v^{2} .
\end{aligned}
$$

Because of the nullity of $F$, the calculation of $H=$ $\sqrt{E G-F^{2}}=\sqrt{E G}$ is easier and yields to

$$
H=\frac{\varepsilon b^{2}(\mu \cos v-a)(\mu-c \cos u)}{(a-c \cos u \cos v)^{2}}
$$

where

$$
\varepsilon=\operatorname{sgn} \text { of }[(\mu \cos v-a)(\mu-c \cos u)] .
$$

Now, the calculation of the different three other coefficients of the second fundamental quadratic form $\varphi_{2}^{M}$ is needed:

$$
\varphi_{2}^{M}(d M)=L d u^{2}+2 M d u d v+N d v^{2}
$$

The calculation of the three components of the vector $(1 / H)(\partial \vec{M} / \partial u) \times(\partial \vec{M} / \partial v)$ is needed for $L, M$, and $N$ so that we obtain at first that

$$
\frac{1}{H} \frac{\partial \vec{M}}{\partial u} \times \frac{\partial \vec{M}}{\partial v}\left(\begin{array}{c}
\varepsilon \frac{(a \cos u \cos v-c)}{A} \\
\varepsilon \frac{b \sin u \cos v}{A} \\
\varepsilon \frac{b \sin v}{A}
\end{array}\right)
$$

where $A=a-c \cos u \cos v$. Then, the second derivatives relating to $u$ and $v$ and both of them read as 


$$
\left.\begin{array}{c}
\frac{\partial^{2} \vec{M}}{\partial u^{2}}\left(\begin{array}{c}
\frac{b^{2}(\mu \cos v-a)(2 a \cos u-3 c \cos v+c \cos 2 u \cos v)}{2 A^{3}} \\
\frac{2 b(\mu \cos v-a)\left(a^{2}+a c \cos u \cos v-2 c^{2}(\cos v)^{2}\right) \sin u}{2 A^{3}} \\
\frac{b c \cos v-a)(2 a \cos u-3 c \cos v+c \cos 2 u \cos v) \sin v}{2 A^{3}}
\end{array}\right), \\
\frac{\partial^{2} \vec{M}}{\partial u \partial v}\left(\begin{array}{c}
\frac{b^{2} \cos u(\mu-c \cos u)\left[a \cos v-c \cos u-c \cos u(\sin v)^{2}\right]}{A^{3}} \\
\frac{b \sin v(\mu-c \cos u)\left[a^{2}-2 c^{2}(\cos u)^{2}+a c \cos u \cos v\right]}{2 A^{3}}
\end{array}\right), \\
\frac{-b c\left[a \cos v-c \cos u-c \cos u(\sin v)^{2}\right]}{A^{3}}
\end{array}\right),
$$

Using (A.8) and (A.9), we obtain

$$
L=\left(\frac{1}{H} \frac{\partial \vec{M}}{\partial u} \times \frac{\partial \vec{M}}{\partial v}\right) \cdot \frac{\partial^{2} M}{\partial u^{2}}=\frac{\varepsilon b^{2}(\mu \cos v-a) \cos v}{A^{2}} .
$$

Using (A.8) and (A.11), we obtain

$$
M=\left(\frac{1}{H} \frac{\partial \vec{M}}{\partial u} \times \frac{\partial \vec{M}}{\partial v}\right) \cdot \frac{\partial^{2} M}{\partial u \partial v}=0 .
$$

Using (A.8) and (A.10), we obtain

$$
N=\left(\frac{1}{H} \frac{\partial \vec{M}}{\partial u} \times \frac{\partial \vec{M}}{\partial v}\right) \cdot \frac{\partial^{2} M}{\partial v^{2}}=\frac{\varepsilon b^{2}(\mu-c \cos u)}{A^{2}} .
$$

The second quadratic fundamental form $\varphi_{2}^{M}(d M)$ reads now as

$$
\begin{aligned}
\varphi_{2}^{M}(d M)= & \frac{\varepsilon b^{2}(\mu \cos v-a) \cos v}{A^{2}} d u^{2} \\
& +\frac{\varepsilon b^{2}(\mu-c \cos u)}{A^{2}} d v^{2} .
\end{aligned}
$$

From [16], the two main curvatures $\rho$ of our Dupin cyclides surfaces at some point $M$ are the solutions of the following equation:

$$
E G \rho^{2}-(L G+E N) \rho+L N=0
$$

Here, the introduction of the different expressions of $E, G$, $L$, and $N$ would be long and clumsy. We therefore calculate the discriminant $\Delta=(L G-E N)^{2}$ of (A.14). When $\Delta>0$, there are two different values for the curvatures of the surface: $\rho_{1}=L / E$ and $\rho_{2}=N / G$. We therefore obtain the following two different main curvature radii (3) and (4) expressed in the core of the paper.

\section{Conflict of Interests}

The authors declare that there is no conflict of interests regarding the publication of this paper.

\section{References}

[1] G. Friedel and F. Grandjean, "Observation geometriques sur les liquidesa coniques fo-cales," Bulletin De La Société Française De Minéralogie, vol. 33, pp. 409-465, 1910.

[2] O. D. Lavrentovich, "Designing Dupin cyclides in micro and macro worlds," Proceedings of the National Academy of Sciences, vol. 110, no. 1, pp. 5-6, 2013.

[3] C. E. Williams, "Helical disclination lines in smectics A," Philosophical Magazine, vol. 32, no. 2, pp. 313-321, 1975.

[4] C. Meyer, Y. A. Nastyshyn, and M. Kleman, "Helical defects in smectic-A and smectic-A* phases," Physical Review E: Statistical, Nonlinear, and Soft Matter Physics, vol. 82, Article ID 031704, 2010.

[5] S. Elston and R. Sambles, The Optics of Thermotropic Liquid Crystals, CRC press, 1998.

[6] G. Friedel, "The mesomorphic states of matter," Annals of Physics, vol. 18, pp. 273-474, 1922. 
[7] M. Kleman and O. D. Lavrentovich, "Grain boundaries and the law of corresponding cones," European Physical Journal E, vol. 2, pp. 47-57, 2000.

[8] I. Dierking, M. Ravnik, E. Lark, J. Healey, G. P. Alexander, and J. M. Yeomans, "Anisotropy in the annihilation dynamics of umbilic defects in nematic liquid crystals," Physical Review E: Statistical, Nonlinear, and Soft Matter Physics, vol. 85, no. 2, Article ID 021703, 2012.

[9] C. Meyer, L. Le Cunff, M. Belloul, and G. Foyart, "Focal conic stacking in smectic a liquid crystals: smectic flower and apollonius tiling," Materials, vol. 2, pp. 499-513, 2009.

[10] O. D. Lavrentovich, "Hierarchy of defect structures in space filling byflexible smectic A layers," Journal of Experimental and Theoretical Physics, vol. 64, pp. 984-990, 1986.

[11] M. Kleman and O. D. Lavrentovich, "Liquids with conics," Liquid Crystals, vol. 36, no. 10-11, pp. 1085-1099, 2009.

[12] J. P. Sethna and M. Kleman, "Spheric domains in smectic liquid crystals," Physical Review A, vol. 26, no. 5, Article ID 3037, 1982.

[13] A. Honglawan, D. A. Beller, M. Cavallaro, R. D. Kamien, K. J. Stebe, and S. Yang, "Pillar-assisted epitaxial assembly of toric focal conic domains of smectic-A liquid crystals," Advanced Materials, vol. 23, no. 46, pp. 5519-5523, 2011.

[14] A. Honglawan, D. A. Beller, M. Cavallaro Jr., R. D. Kamien, and K. J. Stebe, "Topographically-induced hierarchical assembly and geometrical transformation of focal conic domain arrays in smectic liquid crystals," Proceedings of the National Academy of Sciences of the United States of America, vol. 110, pp. 34-39, 2013.

[15] Y. H. Kim, D. K. Yoon, H. S. Jeong, O. D. Lavrentovich, and H.-T. Jung, "Smectic liquid crystal defects for self-assembling of building blocks and their lithographic applications," Advanced Functional Materials, vol. 21, no. 4, pp. 610-627, 2011.

[16] J. Lelong-Ferrand and J. M. Arnaudiès, Cours de Mathématiques, Géométrie Et Cinématique, tome 3, relation 6, Dunod, 2nd edition, 1977. 

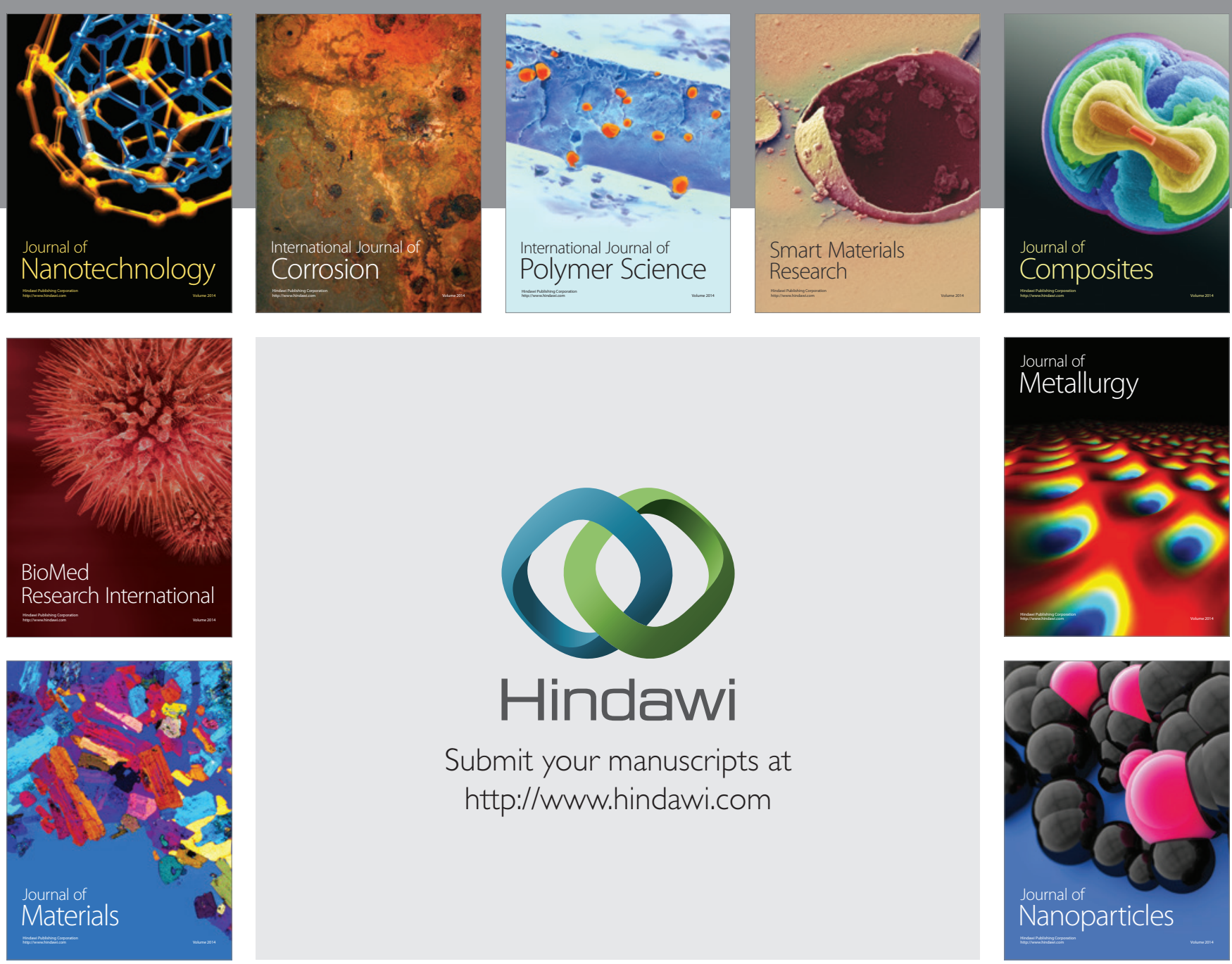

Submit your manuscripts at http://www.hindawi.com
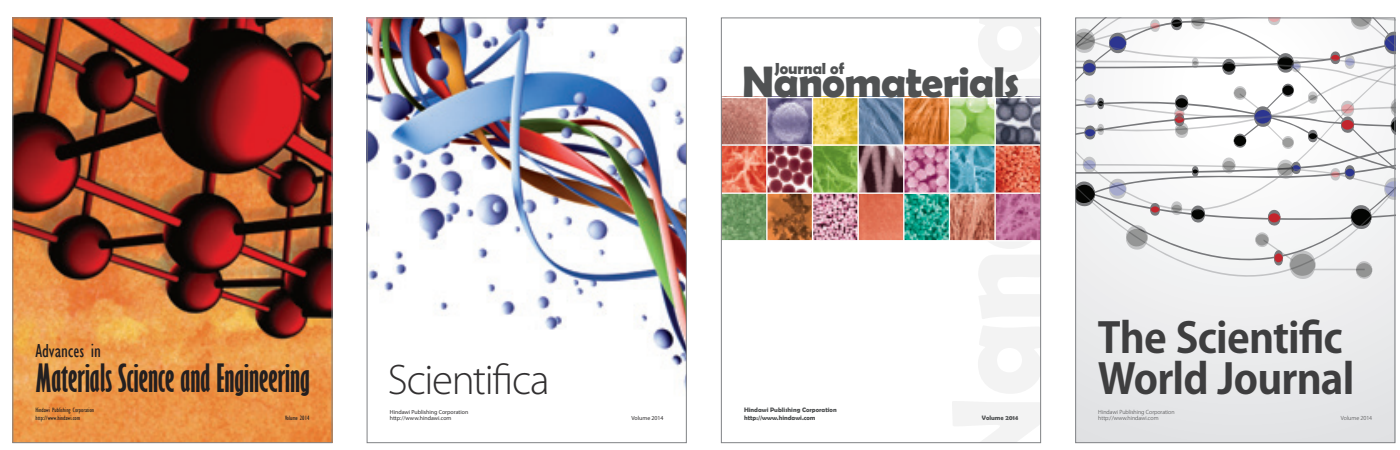

\section{The Scientific World Journal}
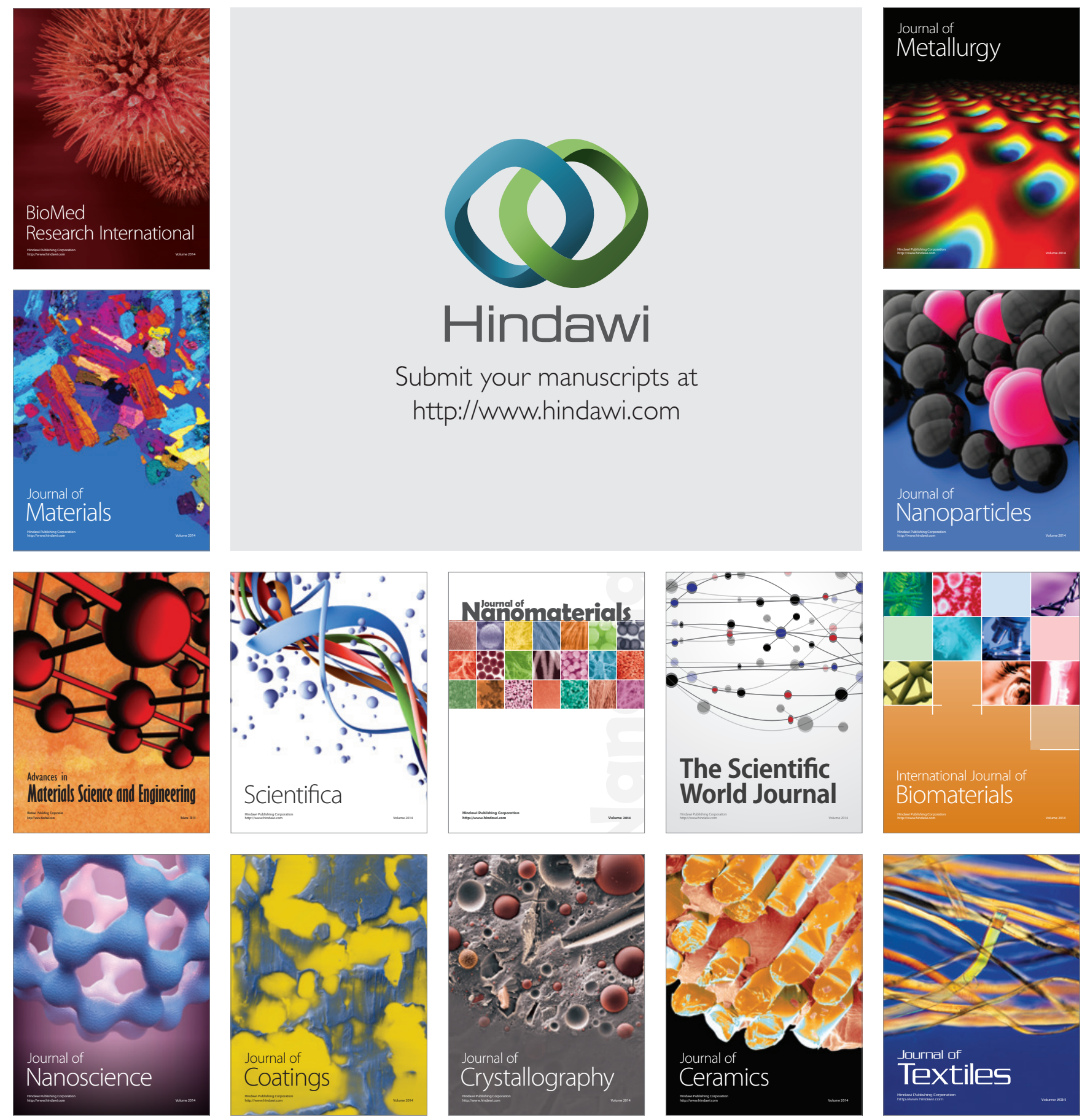